\title{
Delays in Diagnosis and Treatment of Breast Cancer: A Safety-Net Population Profile
}

\author{
Kshama Jaiswal, MDa,b; Madelyne Hull, $\mathrm{MPH}^{\mathrm{c}}$; Anna L. Furniss, $\mathrm{MS}^{\mathrm{d}}$; Reina Doyle, $\mathrm{MPH}^{\mathrm{e}}$; \\ Natalia Gayou, BS ; and Elizabeth Bayliss, MD, MSPH',
}

\begin{abstract}
Background: Timely detection and treatment of breast cancer is important in optimizing survival and minimizing recurrence. Given disparities in breast cancer outcomes based on socioeconomic status, we examined time to diagnosis and treatment in a safety-net hospital. Methods: We conducted a retrospective review of all patients with breast cancer diagnosed between July 1, 2010, and June 30, 2012 $(\mathrm{N}=120)$. We limited our analytic sample to patients with nonrecurrent, primary stage 0 -III breast cancer $(\mathrm{N}=105)$ and determined intervals from presentation to diagnosis, diagnosis to first treatment, last surgery to chemotherapy initiation, and last surgery to start of radiation therapy (RT). Using logistic regression, we calculated unadjusted odds of receiving timely treatment (< median time) versus more delayed treatment ( $\geq$ median time) as a function of age, language, ethnicity, insurance, Charlson comorbidity index, disease stage, method of first presentation (screening mammography vs care provider), symptoms at presentation, and type of surgical treatment. Results: Patients aged 55 to 64 years accounted for most of the sample $(n=37 ; 35.2 \%)$. Median time from presentation to diagnosis ( 23 days), time from diagnosis to first treatment, and time from surgery to chemotherapy initiation fell within intervals published in the literature; median time from last surgery to start of RT was greater than recommended intervals. Factors significantly associated with longer intervals than median time included stage, method of presentation, language, surgical treatment, insurance, and ethnicity. Conclusions: Patients in this safety-net setting experienced acceptable diagnosis and treatment intervals, except for time to RT. Focused interventions that help care providers access imaging quickly for their symptomatic patients could improve time to diagnosis. Concentrating additional efforts on non-English-speaking, Hispanic patients and those who need to receive RT could improve time to treatment.
\end{abstract}

J Natl Compr Canc Netw 2018;16(12):1451-1457 doi: 10.6004/jnccn.2018.7067

Delays in breast cancer care can result in patient dissatisfaction as well as increased risks of local recurrence and death. ${ }^{1-3}$ However, no studies have examined the full continuum of care, from presentation to imaging, to diagnosis, to surgery, to chemotherapy, to radiation therapy (RT). Some studies have examined the time to diagnosis of breast cancer, whereas others have focused on time from diagnosis to surgical treatment. ${ }^{2,4-8}$ Yet others have examined the interval from surgery to chemotherapy, ${ }^{9-13}$ or surgery to RT, ${ }^{1,14-16}$ and a few have ex-

\footnotetext{
aDepartment of Surgery, University of Colorado School of Medicine, Aurora; ${ }^{b}$ Department of Surgery and ${ }^{~}$ Department of Medicine, Denver Health Medical Center, Denver; ${ }^{d}$ ACCORDS at University of Colorado School of Medicine, Aurora; ${ }^{e}$ Health Services Research, Denver Health Medical Center, Denver; ${ }^{f}$ Institute for Health Research, Kaiser Permanente Colorado, Denver; and 'Department of Family Medicine, University of Colorado School of Medicine, Denver, Colorado.

Submitted October 10, 2017; accepted for publication July 31, 2018.

The authors have disclosed that they have no financial interests,
}

amined combinations of these intervals. ${ }^{17-19}$ Our study examines the full continuum of care from presentation through adjuvant RT, as well as the smaller intervals in between.

Most studies of timeliness in breast cancer treatment have been conducted using large cancer registries in the United States, ${ }^{4-6,20-22}$ Canada, ${ }^{2,12,18}$ and Europe. ${ }^{23}$ A few timeliness studies have been performed at institutions with white majority populations. ${ }^{10,19,24}$ Two institutional studies have examined time to treatment in low- arrangements, affiliations, or commercial interests with the manufacturers of any products discussed in this article or their competitors.

Author contributions: Study concept: Jaiswal, Bayliss. Data analysis: Jaiswal. Data entry: Doyle, Gayou. Statistical analysis: Hull, Furniss. Manuscript preparation: Jaiswal. Critical revision: Jaiswal, Hull, Doyle, Gayou, Bayliss.

Correspondence: Kshama Jaiswal, MD, Department of Surgery, Denver Health Medical Center, 777 Bannock Street, MC 0206, Denver, CO 80204. Email: Kshama.Jaiswal@dhha.org 
Jaiswal et al

income populations. ${ }^{7,17}$ However, no studies have examined the full continuum of breast cancer care in an underserved setting. Given racial and ethnic disparities in breast cancer outcomes, it is important to understand processes of care for underserved and minority populations. ${ }^{17}$

This study examines the time intervals from presentation to imaging, diagnosis, to surgery, to chemotherapy, and to RT in a safety-net population with breast cancer. We examined the association between time intervals and socioeconomic factors such as language, ethnicity, and insurance. We also assessed the relationship between time intervals and patient medical factors such as disease stage, symptoms at presentation, and surgical management with lumpectomy or mastectomy.

\section{Methods}

\section{Study Design and Setting}

We conducted a retrospective cohort analysis of patients receiving breast cancer care at Denver Health and Hospital Authority (DH), which is an integrated, safety-net hospital system that serves Denver and the surrounding counties.

\section{Study Sample}

Our initial cohort consisted of all patients who received any care for breast cancer $(\mathrm{N}=120)$ from July 1, 2010, through June 30, 2012. Patients were identified by the hospital-based cancer registry. We excluded patients with primary stage IV disease or missing staging data $(n=11)$ because they have a different treatment trajectory than those with stage 0-III disease. We also excluded patients with recurrent breast cancer within 5 years of primary diagnosis $(n=2)$ because of the possibility that they were already under the care of an oncologist, and therefore their timeliness of care was not comparable to those who were newly diagnosed. An additional 2 patients who had both stage IV disease and recurrent cancer were excluded. The remaining 105 patients comprised our analytic study sample.

\section{Data Collection}

Data were abstracted from electronic health records and entered into a REDCap database. Date of diagnosis was considered the date of the diagnostic biopsy. Data elements included intervals between presentation to diagnosis, diagnosis to first treatment, last surgery to chemotherapy start, and last surgery to RT start. We also stratified specific steps within these larger intervals. In the interval from presentation to diagnosis, we measured the time from presentation to first imaging, first imaging to last imaging before diagnostic biopsy, and last imaging to diagnostic biopsy. In the interval from diagnosis to first treatment, we measured the time from diagnostic biopsy to the time when the patient was notified of their diagnosis, their first visit with an oncology clinic (either surgical or medical), and the time to first treatment. Date of first treatment was defined as either the date of the first surgery or the first date of neoadjuvant chemotherapy.

\section{Data Analysis}

We defined presentation as either symptomatic (ie, patient had a primary care or urgent care visit with specific breast complaints, such as mass, discharge, pain) or asymptomatic (ie, patient had an abnormal screening mammogram). If screening mammography was the method of presentation, the first image was defined as the date of presentation. Underinsured patients were defined as those with coverage through CICP (Colorado Indigent Care Program; a state-funded program) or DFAP (Denver Health Financial Assistance Program; a hospital-based charity program), or were self-pay. Timely treatment occurred at less than the median time, whereas delayed treatment occurred at or later than the median time. The limited number of patients in our study did not allow a more nuanced comparison of the fastest versus slowest quartile.

All calculated intervals were limited to events occurring at $\mathrm{DH}$ or at contracted neighboring hospitals for RT. As a safety-net hospital, patients may enter and exit the hospital system at all points, resulting in interrupted care. For example, a patient may come to $\mathrm{DH}$ after completion of diagnostic workup or may have the diagnostic biopsy performed at $\mathrm{DH}$ but then choose to go elsewhere for treatment.

We determined the median time for all intervals. We used logistic regression to calculate unadjusted odds of receiving timely treatment as a function of age, language, ethnicity, insurance, Charlson comorbidity index, disease stage, context of first presentation (screening mammography vs care 
provider), symptoms at presentation, and type of surgical treatment.

\section{Results}

A total of 62 patients $(59.0 \%)$ were aged $\geq 55$ years. Most patients were of Hispanic origin (43.8\%), 80 (76.2\%) spoke English, and 31 (29.5\%) were underinsured. Of the 74 people who were insured, 32 (43.2\%) were on Medicaid (Table 1 ).

Among patients who presented with breast cancer, underwent imaging, and were diagnosed at $\mathrm{DH}$ $(n=97)$, the median time from presentation to diagnosis was 23 days (Figure 1). For most patients, screening mammography was the method of pre-

\begin{tabular}{|c|c|c|}
\hline & $\begin{array}{c}n \\
(N=105)\end{array}$ & (\%) \\
\hline \multicolumn{3}{|l|}{ Age, y } \\
\hline$<45$ & 16 & (15.2) \\
\hline 45 to $<55$ & 27 & (25.7) \\
\hline 55 to $<65$ & 37 & $(35.2)$ \\
\hline$\geq 65$ & 25 & (23.8) \\
\hline \multicolumn{3}{|l|}{ Race/Ethnicity } \\
\hline Hispanic/Not black & 46 & (43.8) \\
\hline White/Not Hispanic & 30 & (28.6) \\
\hline Black & 25 & (23.8) \\
\hline Other & 4 & (3.8) \\
\hline \multicolumn{3}{|l|}{ Primary language } \\
\hline English & 80 & (76.2) \\
\hline Spanish & 17 & $(16.2)$ \\
\hline Other & 8 & (7.6) \\
\hline \multicolumn{3}{|c|}{ Insurance status at time of diagnosis } \\
\hline Medicaid & 32 & (30.5) \\
\hline Medicare & 28 & (26.7) \\
\hline $\mathrm{CICP}$ & 19 & $(18.1)$ \\
\hline Commercial & 14 & (13.3) \\
\hline DFAP/Other & 12 & (11.4) \\
\hline \multicolumn{3}{|c|}{ Charlson comorbidity index } \\
\hline 0 & 31 & (29.5) \\
\hline 1 & 24 & (22.9) \\
\hline 2 & 21 & (20.0) \\
\hline$\geq 3$ & 29 & (27.8) \\
\hline \multicolumn{3}{|c|}{ Symptomatic at presentation } \\
\hline Yes & 41 & (39.1) \\
\hline \multicolumn{3}{|c|}{ Disease stage at presentation } \\
\hline 0 & 22 & $(21.0)$ \\
\hline I & 32 & (30.5) \\
\hline II & 42 & $(40.0)$ \\
\hline III & 9 & (8.6) \\
\hline \multicolumn{3}{|l|}{ Surgical management } \\
\hline Lumpectomy & 49 & (46.7) \\
\hline Mastectomy & 42 & $(40.0)$ \\
\hline No surgery at $\mathrm{DH}$ & 14 & $(13.3)$ \\
\hline
\end{tabular}

Abbreviations: $\mathrm{CICP}$, Colorado Indigent Care Program; DFAP, Denver Health Financial Assistance Program; DH, Denver Health and Hospital Authority.

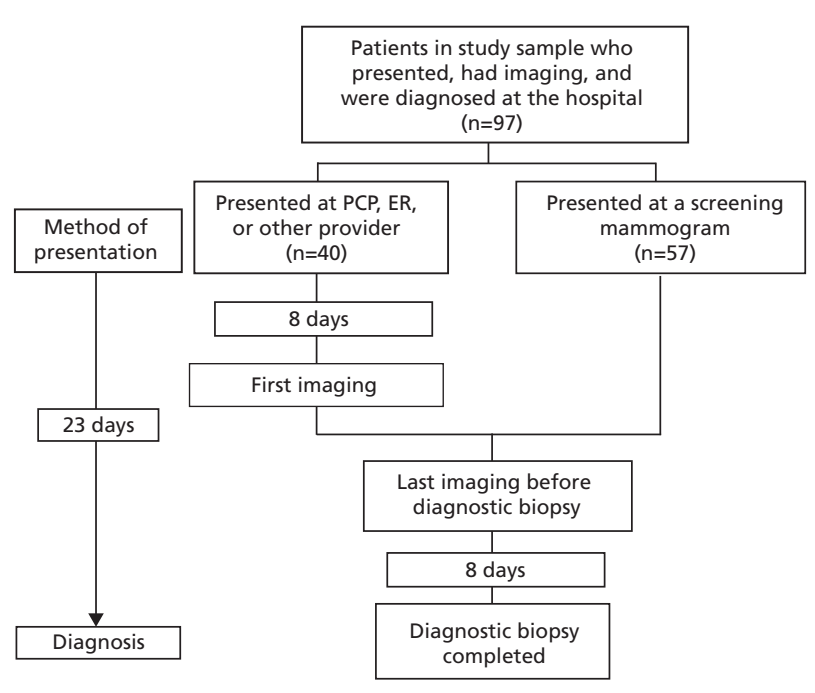

Figure 1. The time to diagnosis and treatment, with interval expressed in median days.

Abbreviations: ER, emergency room; PCP, primary care physician.

sentation $(n=57 ; 58.8 \%)$. If a patient presented to a primary care physician, emergency room, or other provider $(n=40)$, a median of 8 days was needed to obtain the first image. Approximately half of the patients $(n=55)$ needed additional imaging before proceeding to diagnostic biopsy. For these patients, the median time from first imaging to last imaging before biopsy was 27 days. When it was determined that a patient needed a biopsy, a median of 8 days was required to obtain the procedure.

Among patients who were diagnosed and treated at $\mathrm{DH}(\mathrm{n}=91)$, the median time from diagnosis to first treatment was 37 days (Figure 2). Once the patient underwent a diagnostic biopsy, it took a median of 8 days to notify them of the result and an additional 8 days to be seen in the surgical or medical oncology clinic. Once evaluated in the clinic, a median of 16 days was needed for the patient to undergo initial treatment. Most patients $(n=88 ; 96.7 \%)$ received surgery as their first treatment, whereas 3 (3.3\%) had chemotherapy.

Patients experienced different intervals between treatments depending on their treatment path. The average transition time from surgery to the medical oncology clinic was 13 days. Of the 84 patients who went on to visit the medical oncology clinic, 33 (39.3\%) received chemotherapy. Median time from last surgery to chemotherapy initiation was 48 days. For patients needing surgery and RT only, without intervening chemotherapy $(n=29)$, median time 
Jaiswal et al

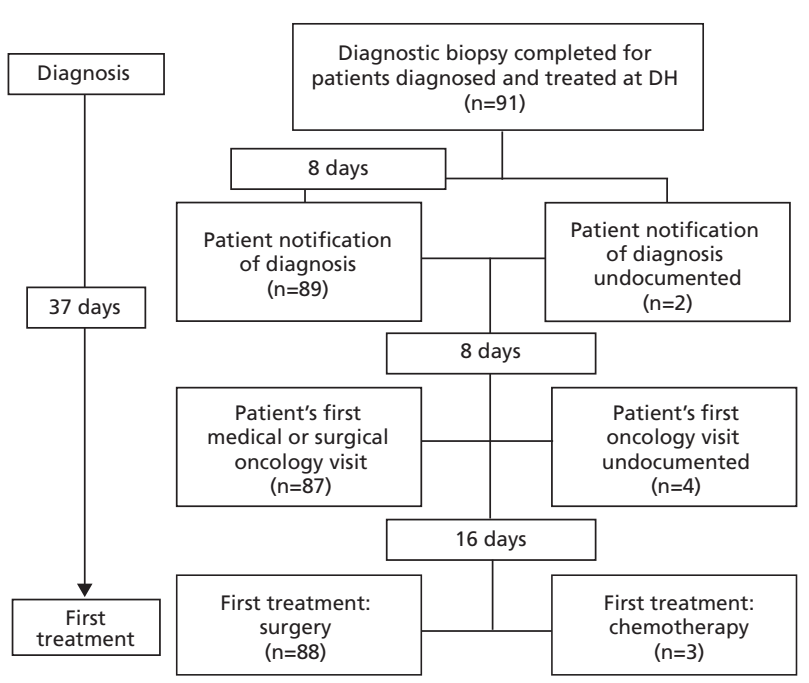

Figure 2. Time to treatment.

Abbreviation: DH, Denver Health and Hospital Authority.

from last surgery to first radiation treatment was 69 days (Table 2). For patients with an available date of radiation oncology referral $(n=45)$, median time from referral to evaluation in the radiation oncology clinic was 22 days. For patients electing RT $(n=53)$, median time from radiation oncology evaluation to first radiation treatment was 16 days.

A number of patient characteristics were found to significantly impact a patient's odds of timely care. As Table 3 illustrates, patients with stage II or III breast cancer (odds ratio [OR], 2.32; 95\% CI, 1.045.15 ) or who presented to a care provider (OR, 3.03; 95\% CI, 1.33-6.89) were more likely to experience longer intervals between presentation and diagnosis. Patients who spoke English as their primary language (OR, 0.20; 95\% CI, 0.07-0.61) experienced a

\begin{tabular}{|lccc|}
\hline \multicolumn{3}{|l|}{ Table 2. Intervals of Care } \\
\hline Interval & Median (d) & $\begin{array}{c}\text { 25th, 75th } \\
\text { Percentile (d) }\end{array}$ & $\begin{array}{c}\text { Recommended Interval in } \\
\text { Published Literature (d) }\end{array}$ \\
\hline $\begin{array}{l}\text { Presentation } \\
\text { to diagnosis }\end{array}$ & 23 & 11,46 & $60^{22}$ \\
\hline $\begin{array}{l}\text { Diagnosis to } \\
\text { first treatment }\end{array}$ & 37 & 29,49 & $21-60^{4,7,18,22}$ \\
\hline $\begin{array}{l}\text { Presentation to } \\
\text { first treatment }\end{array}$ & 65 & 52,87 & $\mathrm{~N} / \mathrm{A}$ \\
\hline $\begin{array}{l}\text { Last surgery to } \\
\text { chemotherapy } \\
\text { initiation }\end{array}$ & 48 & 31,63 & $28-90^{9-13}$ \\
\hline $\begin{array}{l}\text { Last surgery } \\
\text { to radiation } \\
\text { start }\end{array}$ & 69 & 53,88 & $42-56^{1,14-16}$ \\
\hline
\end{tabular}

Abbreviation: N/A, not applicable.

${ }^{a}$ For patients needing surgery and radiation only, without intervening chemotherapy. shorter time from diagnosis to first treatment. However, patients who underwent breast conservation surgery had greater odds (OR, 2.48; 95\% CI, 1.065.80) of experiencing longer intervals from diagnosis to first treatment. Presentation to a care provider (OR, 2.92; 95\% CI, 1.20-7.10), Hispanic ethnicity (OR, 3.38; 95\% CI, 1.41-8.12), and being underinsured (OR, 4.38; 95\% CI, 1.53-12.53) all significantly increased the odds of a patient experiencing longer intervals between presentation and first treatment.

\section{Discussion}

In 2001, the Institute of Medicine identified timeliness of care as 1 of 6 measures of quality. ${ }^{25}$ Time to diagnosis and to treatment of breast cancer has become the subject of accreditation and quality control agencies, such the National Quality Measures for Breast Centers (NQMBC), ${ }^{26}$ ASCO, and NCCN. ${ }^{27}$ However there is no consensus on what time frame constitutes a delay.

Causes of longer intervals between diagnosis and treatment are clearly multifactorial; patient factors, symptomatology, treatment factors, and hospital system factors may all play a role. Our study found that patient factors such as being underinsured, Hispanic, and non-English-speaking all were associated with delays greater than the median time. This is similar to findings in the SEER population and the National Cancer Database, which showed that Hispanic and African American patients experienced diagnostic and treatment delays., ${ }^{4,6}$ Although our study found an unadjusted OR of 4.4 for underinsured patients experiencing delay to first treatment, a study from another safety-net hospital found that only African American race (OR, 2.7) was associated with treatment delay; however, this study did not specifically examine insurance status. ${ }^{28}$ A US comprehensive cancer center also found that women with $\geq 5$ risk factors, including non-White or Hispanic race, breast symptoms, and far distance from cancer center, had a 12 -fold risk of diagnostic delay. ${ }^{29}$

In our study, patients' disease stage, method of presentation, and symptomatology are likely related. Patients with later stage of disease (stage II or III) were more likely to experience delay in diagnosis. This is likely due to the fact that patients with laterstage disease often present to a provider with symptoms, who must then order imaging, rather than 
Delays in Diagnosis and Treatment of Breast Cancer

\begin{tabular}{|c|c|c|c|c|}
\hline Interval & Variable & Reference & OR & $P$ Value \\
\hline \multirow[t]{2}{*}{ Presentation to diagnosis } & Stage II or III & Stage 0 or 1 & 2.32 & .0395 \\
\hline & Method of presentation: care provider & Method of presentation: screening mammogram & 3.03 & .0083 \\
\hline \multirow[t]{4}{*}{ Diagnosis to first treatment } & English-speaking & Non-English-speaking & 0.20 & .0045 \\
\hline & Symptomatic & Nonsymptomatic & 2.22 & .0700 \\
\hline & Lumpectomy & Mastectomy & 2.48 & .0300 \\
\hline & $\mathrm{CCl} \geq 3$ & $\mathrm{CCl}<3$ & 0.59 & .2700 \\
\hline \multirow[t]{3}{*}{ Presentation to first treatment } & Underinsured & Insured & 4.38 & .0058 \\
\hline & Hispanic & Non-Hispanic & 3.38 & .0063 \\
\hline & Method of presentation: care provider & Method of presentation: screening mammogram & 2.92 & .0101 \\
\hline
\end{tabular}

Abbreviations: $\mathrm{CCl}$, Charlson comorbidity index; OR, odds ratio.

presenting directly for imaging; patients who presented to a care provider had 3 times the odds of experiencing a delay in diagnosis compared with those who presented directly for imaging. Our study also demonstrated that patients who were symptomatic at presentation were more likely to experience a delay, but this did not achieve statistical significance. This is in contrast to findings in other safety-net systems, where method of presentation, symptomatology, or late-stage disease were not associated with diagnostic delay. ${ }^{28}$ However, an Italian study with a relatively large number of symptomatic patients $(79 \%)$ found that patients with symptomatic, self-detected cancer had 2 times the odds of experiencing a delay in diagnosis relative to patients with mammogram-detected cancer. $^{30}$

This study highlights the need for hospital system process improvements. Part of the diagnostic delay may be the process of ordering and scheduling mammography after being seen by a provider. Arranging imaging may not occur while the patient is physically still in clinic, which could explain the delay. This initial delay also affects the time to first treatment. Patients who presented to a care provider had approximately 3 times the odds of taking longer than the median to start treatment. Development of standard care pathways through the emergency room, urgent care, and primary care could impact these delays. Additionally, nurse navigation programs, which have been shown to improve adherence to guidelineconcordant breast cancer care in safety-net hospital systems, ${ }^{31}$ may also improve timeliness.

Process improvements in radiology may also be required. Approximately half of the patients needed additional imaging beyond initial studies, and these patients experienced delays between first and last imaging, with a median of 27 days for the complete radiologic evaluation prior to biopsy. The reasons for this could be multifactorial, and may include patient preference or limitations in radiologic scheduling. Further examination of imaging availability is warranted.

In the time from diagnostic biopsy to first treatment, the median of 8 days for the patient to be notified of the results plus an additional 8 days to be seen in an oncology clinic contributed significantly to the overall median 37 days to starting treatment. Process improvements in the radiology department and improved access to the oncology clinics could significantly reduce this delay.

Treatment factors can also affect timeliness. In our study, patients who underwent lumpectomy took longer to start their first treatment, potentially because of additional workup required to evaluate disease extent and to ensure that they were adequate candidates for lumpectomy. Although many would expect that patients undergoing mastectomy would have a longer time to first treatment, potentially due to more advanced disease that requires imaging for staging prior to surgery or due to patient preference for reconstruction, our study did not demonstrate this to be the case. Patients who underwent neoadjuvant therapy $(n=3)$ did so to facilitate mastectomy rather than breast-conserving surgery. Treatment delays $>12$ weeks have been shown to have adverse survival outcomes compared with treatment within $<4$ weeks of diagnosis. ${ }^{32}$ Median time to first treatment in our cohort was 5.2 weeks.

The most apparent treatment delay attributed to our hospital system is the delay to RT treatment. With radiation oncology services contracted with neighboring hospitals, our patients have a median time of 69 days to starting RT after their last surgery when no other adjuvant chemotherapy was indicat- 
Jaiswal et al

ed. The process of external referral to radiation oncology adds significantly to the delay (median of 22 days from referral to being seen in radiation clinic), but does not explain all of the delay. Another potential source of delay is the fact that during the study time frame, radiation referral was not issued by surgeons in the postoperative period. Instead, patients were sent from surgeons to the medical oncology clinic, who would then issue radiation referral. This is particularly concerning because delays beyond 6 to 8 weeks have been associated with an increase in local recurrence rates, particularly for patients undergoing breast-conserving therapy. ${ }^{14,16,33}$ Other studies have shown that when radiation services are located farther away, patients are more likely to undergo mastectomy, suggesting that the distance to the radiation treatment facility could be a barrier to successful breast-conserving therapy. ${ }^{34}$ This finding clearly highlights the need for more timely referrals to radiation services and/or locating radiation services closer to the main campus of our hospital.

Our study has several limitations. It is a singleinstitution, retrospective study with a relatively small sample size, with data from 2010 to 2012. This initial study is the baseline for our current studies, which are continuing to examine the timeliness of more recent populations of patients. Additionally, as a quantitative study, we lack the informative details that a qualitative analysis can bring. Our future studies will need to incorporate key informant interviews to better understand why some populations experience delays. An additional limitation of our data- base is that we are unable to capture a physician's recommended treatment relative to whether the patient received it. For future studies, we would like to capture the provider recommendation as well as whether the patient was able to follow through with that recommendation. Lack of adherence to recommendations may also provide an important insight into delays experienced by our patients.

The strengths of our study include the safety-net hospital setting in which it was conducted. Funding at safety-net hospitals for breast cancer quality improvement efforts is limited, and therefore there are few published studies from safety-net systems. This study brings attention to vulnerable populations and sought to understand delays in their care so they are not further disadvantaged. This study also examined the entire continuum of breast cancer from presentation to RT as well as many smaller intervals within the continuum.

\section{Conclusions}

Our study has elucidated patient and system factors affecting the timeliness of care at a safety-net hospital. Programmatic changes that could enhance efficiency of obtaining imaging among symptomatic patients and accessibility to subsequent diagnostic imaging could improve time to diagnosis. Concentrating efforts on non-English-speaking, Hispanic patients and those who need RT could improve time to treatment.

\section{References}

1. Desai S, Hurley J, Takita $\mathrm{C}$, et al. Impact of surgery-radiation interval on locoregional outcome in patients receiving neo-adjuvant therapy and mastectomy. Breast J 2013;19:427-430.

2. Cordeiro E, Dixon M, Coburn N, Holloway CM. A patient-centered approach to wait times in the surgical management of breast cancer in the province of Ontario. Ann Surg Oncol 2015;22:2509-2516.

3. Bleicher RJ, Ruth K, Sigurdson ER, et al. Time to surgery and breast cancer survival in the United States. JAMA Oncol 2016;2:330-339.

4. Bilimoria KY, Ko CY, Tomlinson JS, et al. Wait times for cancer surgery in the United States: trends and predictors of delays. Ann Surg 2011;253:779-785

5. Bleicher RJ, Ruth K, Sigurdson ER, et al. Time to surgery and breast cancer survival in the United States. JAMA Oncol 2016;2:330-339.

6. Bleicher RJ, Ruth K, Sigurdson ER, et al. Preoperative delays in the US Medicare population with breast cancer. J Clin Oncol 2012;30:4485-4492.

7. Brazda A, Estroff J, Euhus D, et al. Delays in time to treatment and survival impact in breast cancer. Ann Surg Oncol 2010;17(Suppl 3):291-296.

8. Yoo TK, Han W, Moon HG, et al. Delay of treatment initiation does not adversely affect survival outcome in breast cancer. Cancer Res Treat 2016;48:962-969.
9. Fedewa SA, Ward EM, Stewart AK, Edge SB. Delays in adjuvan chemotherapy treatment among patients with breast cancer are more likely in African American and Hispanic populations: a national cohort study 2004-2006. J Clin Oncol 2010;28:4135-4141.

10. Gagliato Dde M, Gonzalez-Angulo AM, Lei X, et al. Clinical impact of delaying initiation of adjuvant chemotherapy in patients with breast cancer. J Clin Oncol 2014;32:735-744.

11. Nurgalieva ZZ, Franzini L, Morgan RO, et al. Impact of timing of adjuvant chemotherapy initiation and completion after surgery on racial disparities in survival among women with breast cancer. Med Oncol 2013;30:419.

12. Vandergrift JL, Niland JC, Theriault RL, et al. Time to adjuvant chemotherapy for breast cancer in National Comprehensive Cancer Network institutions. J Natl Cancer Inst 2013;105:104-112.

13. Bunnell CA, Losk K, Kadish S, et al. Measuring opportunities to improve timeliness of breast cancer care at Dana-Farber/Brigham and Women's Cancer Center. J Natl Compr Canc Netw 2014;12(Suppl 1):S5-9.

14. Huang J, Barbera $L$, Brouwers $M$, et al. Does delay in starting treatment affect the outcomes of radiotherapy? A systematic review. J Clin Oncol 2003;21:555-563.

15. Jobsen JJ, van der Palen J, Baum M, et al. Timing of radiotherapy in breastconserving therapy: a large prospective cohort study of node-negative breast cancer patients without adjuvant systemic therapy. Br J Cancer 2013;108:820-825. 
Delays in Diagnosis and Treatment of Breast Cancer

16. Punglia RS, Saito AM, Neville BA, et al. Impact of interval from breast conserving surgery to radiotherapy on local recurrence in older women with breast cancer: retrospective cohort analysis. BMJ 2010;340:c845.

17. McLaughlin JM, Anderson RT, Ferketich AK, et al. Effect on survival of longer intervals between confirmed diagnosis and treatment initiation among low-income women with breast cancer. J Clin Oncol 2012;30:44934500 .

18. Gorey KM, Luginaah IN, Holowaty EJ, et al. Wait times for surgical and adjuvant radiation treatment of breast cancer in Canada and the United States: greater socioeconomic inequity in America. Clin Invest Med 2009;32:E239-249.

19. Golshan M, Losk K, Kadish S, et al. Understanding process-of-care delays in surgical treatment of breast cancer at a comprehensive cancer center Breast Cancer Res Treat 2014;148:125-133.

20. Ahmed MM, Piper JM, Hansen NA, et al. Novel magnet-retained prosthetic system for facial reconstruction. JAMA Facial Plast Surg 2014;16:55-57.

21. Miller JW, Hanson V, Johnson GD, et al. From cancer screening to treatment: service delivery and referral in the National Breast and Cervical Cancer Early Detection Program. Cancer 2014;120(Suppl 16):2549-2556

22. Richardson LC, Royalty J, Howe W, et al. Timeliness of breast cancer diagnosis and initiation of treatment in the National Breast and Cervical Cancer Early Detection Program, 1996-2005. Am J Public Health 2010;100:1769-1776

23. Torring ML, Frydenberg M, Hansen RP, et al. Evidence of increasing mortality with longer diagnostic intervals for five common cancers: a cohort study in primary care. Eur J Cancer 2013;49:2187-2198.

24. Landercasper J, Linebarger JH, Ellis RL, et al. A quality review of the timeliness of breast cancer diagnosis and treatment in an integrated breast center. J Am Coll Surg 2010;210:449-455.
25. Institute of Medicine Committee on Quality of Health Care in America. Crossing the Quality Chasm: A New Health System for the 21st Century. Washington, DC: National Academy Press; 2001.

26. Kaufman CS, Shockney L, Rabinowitz B, et al. National Quality Measures for Breast Centers (NQMBC): a robust quality tool: breast center quality measures. Ann Surg Oncol 2010;17:377-385.

27. Desch CE, McNiff KK, Schneider EC, et al. American Society of Clinical Oncology/National Comprehensive Cancer Network quality measures. J Clin Oncol 2008;26:3631-3637.

28. Williams DL, Tortu S, Thomson J. Factors associated with delays to diagnosis and treatment of breast cancer in women in a Louisiana urban safety net hospital. Women Health 2010;50:705-718.

29. Stuver SO, Zhu J, Simchowitz B, et al. Identifying women at risk of delayed breast cancer diagnosis. Jt Comm J Qual Patient Saf 2011;37:568-575.

30. Crispo A, Montella M, Barba M, et al. Association between mode of breast cancer detection and diagnosis delay. Breast 2009;18:382-386.

31. Rizzo M, Bumpers J, Okoli J, et al. Improving on national quality indicators of breast cancer care in a large public hospital as a means to decrease disparities for African American women. Ann Surg Oncol 2011;18:34-39.

32. Polverini AC, Nelson RA, Marcinkowski E, et al. Time to treatment: measuring quality breast cancer care. Ann Surg Oncol 2016;23:3392-3402.

33. Tsoutsou PG, Koukourakis MI, Azria D, Belkacémi Y. Optimal timing for adjuvant radiation therapy in breast cancer: a comprehensive review and perspectives. Crit Rev Oncol Hematol 2009;71:102-116.

34. Goyal S, Chandwani S, Haffty BG, Demissie K. Effect of travel distance and time to radiotherapy on likelihood of receiving mastectomy. Ann Surg Oncol 2015;22:1095-1101.

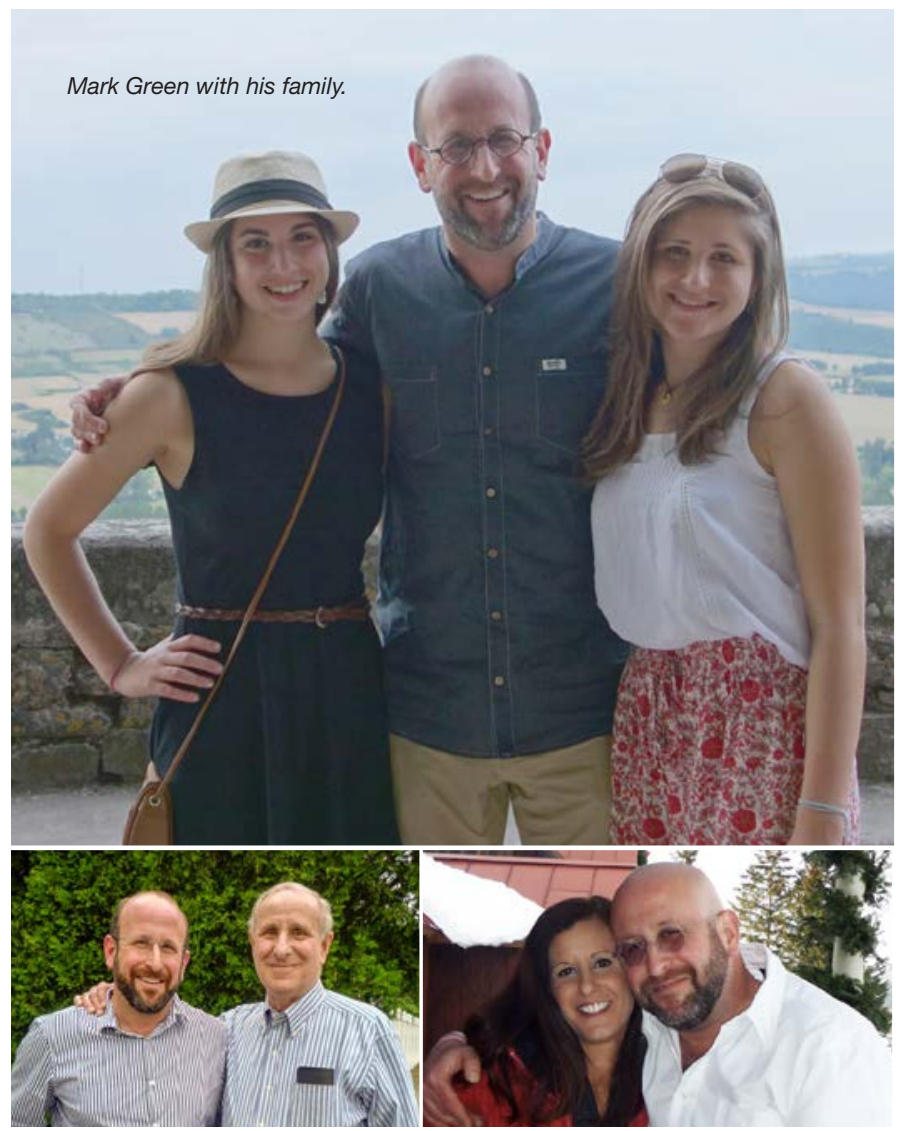

\section{Support families facing cancer together.}

For the Green Family and the 1.7 million people diagnosed with cancer in the U.S. each year, NCCN Foundation ${ }^{\circledR}$ has pledged to update and add to the library of NCCN Guidelines for Patients ${ }^{\circledR}$.

Your support enables us to provide up-to-date, trustworthy information to families like Mark's, empowers them to make the right choices about care, and helps to ensure the best possible outcomes for every patient.
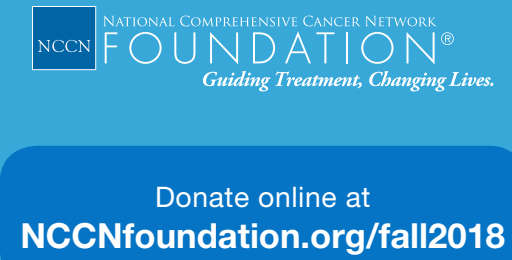